\title{
How Does the Level of Reservoir Description Impact the Choice of Development / EOR Process?
}

\author{
Damsleth E., Wilcox L., Farrow G., Rodgers S.
}

Norsk Hydro E \& P , Oslo, Norway

\begin{abstract}
Copyright 1995, Steering Committee of the European IOR - Symposium.
This paper was presented at the Bth. European IOR - Symposium in Vienna, Austria, May 16 - 17, 1995

This paper was selected for presentation by the Steening Committee, following review of information contained in an abstract submitted by the author(s). The paper, as presented hes not been reviewed by the Steering Committee.
\end{abstract}

\begin{abstract}
Differences in recovery under several displacement mechanisms. for successive increases in reservoir detail, are outlined for the Brent-NI reservoir in the Visund field off Western Norway.
\end{abstract}

The evaluation progressed from an early comparative study conducted on a 2-D layer cake model through a 3-D layer cake model to a reservoir description based on petrophysically defined lithofacies, which allowed for vertical and lateral reservoir heterogeneity. Finally the deterministic heterogeneous model was tested by introducing stochastically distributed reservoir properties that were conditioned to the lithofacies description. In addition to the level of reservoir heterogeneity, the $\mathrm{Vi}$ sund Brent-NI dual fluid system necessitated the evaluation to be expanded from a black-oil (immiscible) to a compositional (miscible) simulation study.

The case study shows that the choice of development/ EOR process depends critically on: (i) The level of detail in the reservoir description, (ii) treatment of the fluid property behaviour, and (iii) aggressive well interventions. Straightforward $2-D$ and equal interval layercake models give similar, over-optimistic results, both for black-oil and compositional simulation, while the results in the more heterogeneous models are significantly dependent on the type of simulation. Under compositional simulation the recovery was reduced for the EOR schemes: the improved local displacement efficiency (miscibility) was found to adversely affect the vertical sweep (gravity segregation). When studying heterogeneous models. an aggressive well intervention program may prove more practical and cost effective than EOR schemes.

\section{Introduction}

Incorporating static reservoir information into a representative model to predict realistic future dynamic behaviour, is a technically demanding exercise. Experience from producing fields is riddled with accounts of unexpected problems that significantly changed the actual reservoir performance from earlier predictions. Vertical and lateral reservoir continuity are two of the main culprits in the underperformance of several known Brent Group reservoirs ${ }^{1-3}$, although predicted reservoir quality and aquifer support have also contributed significantly ${ }^{4}$.

New tools are providing various means of gaining a more truthful reservoir description. Geophysical interpretations made with 3-D seismic technology along with lineament and azimuth analysis are providing enhanced interpretations of faulting and consequently reservoir compartmentalization. This technology however can only be taken to the point of its resolution beyond which dynamic data must assist with the analysis. Stochastic modelling of reservoir bodies, parameters and subseismic faulting are also tools that have gained prominence in filling in the uncertainty in our perception of the "true" reservoir description ${ }^{5,6}$. It must be emphasized that these tools, including the use of producing field analogues, only become valuable when they are used by integrated teams of geoscientists and engineers.

Evaluating EOR schemes frequently necessitates screening studies to be completed on detailed 2-D cross-sections or small segment modeis. The results of these studies often establish the preferred displacement mechanism. which is then carried forward to full field reservoir modelling. Incorporation of reservoir detail is however difficult in these smaller models especially vertical connectivity and lateral variation.

The following evaluation details the challenges of determining the technically optimal recovery mechanism. A case study is presented which follows the chronological development of the Brent-NI reservoir in the Visund field. The initial detailed 2-D model was expanded to a traditional 3-D "layer-cake" model, which in turn was 


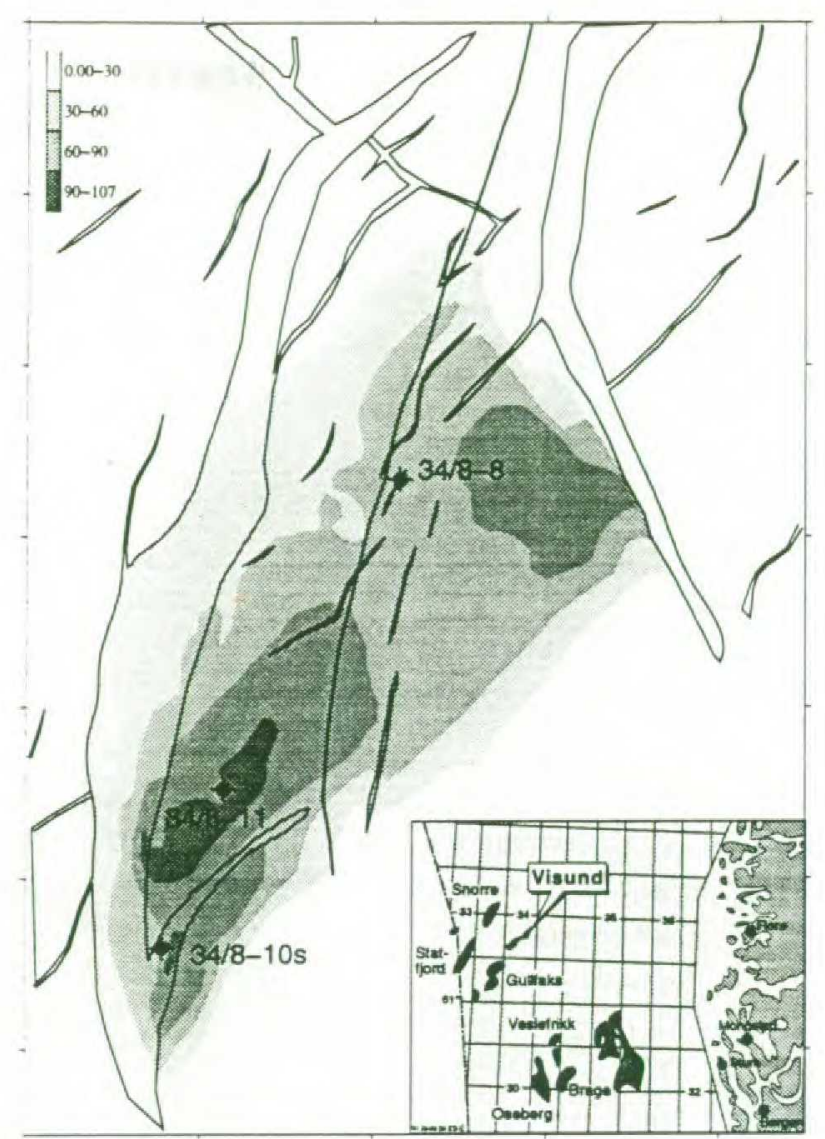

Figure 1. Visund Brent-NI location and net hydrocarbon column map.

recognized to lack the vertical and lateral heterogeneity that has been observed in other producing Brent Group reservoirs. A further enhancement was made to the reservoir description based on petrophysically defined lithofacies, which allowed for vertical and lateral reservoir heterogeneity. Finally the deterministic heterogeneous model was tested by introducing stochastically distributed reservoir properties that were conditioned to the lithofacies description.

The dual-fluid system discovered in Brent-NI was found to contain "black-oil" and "slightly-volatile-oil" fluid types. The evaluation therefore necessitated the use of compositional simulation in order to account for the favourable mass exchange with the volatile oil fluid system. In addition it was recognized that fluid crossflow and gravity segregation would play important roles in the recovery and would therefore require proper modelling through the use of a compositional simulator ${ }^{7}$. The analysis also shows a reference "black-oil" simulation, to illustrate the importance, in addition to the reservoir description detail, of the reservoir fluid property performance.

\section{Reservoir Description}

\subsection{Structural Model and Hydrocarbon Filling History}

The Visund field is a tilted fault-block hydrocarbon accumulation located $20 \mathrm{~km}$ SE of the Snorre field and 15 $\mathrm{km}$ NE of Gullfaks, but significantly downthrown with respect to them both (Figure 1).
Visund is sharply bounded along its southeastern edge by the Visund Fault, the major graben-bounding fault to the North Viking Graben. It's NW limit is gradational into the Pancake Basin, while the southerly limit is much sharper: a structural saddle at top-Brent level represents a palaeo-spillpoint from Visund to Gullfaks.

Hydrocarbons have been discovered in the Lunde and Statfjord formations and in the Brent Group. The filling history has been a turbulent one, and present-day hydrocarbon distribution is a mere shadow of a once giant oil accumulation, most of which leaked during Miocene uplift, erosion and reduced caprock integrity.

\section{$\underline{2.2 \text { Sedimentology }}$}

The Visund area lies close to the northern limit of the Brent delta system, and in such a palaeogeographic setting the reservoir shows considerable three-dimensional heterogeneity ${ }^{8}$.

The Visund reservoir was divided into 7 zones according to sequence stratigraphic principles using the complete core database. The two lowermost zones, $1 \mathrm{~B}$ and $1 C$, consist of classical Rannoch wave-dominated laminated micaceous sandstones. Zone 2 (I.Etive) is distinguished by the incoming of sharp-based pulses of coarser sands reflecting fluvial input, variably modified by wave and tidal processes, which reached its peak at the base of zone $3 A$ (u. Etive). Zone $3 B$ (Ness) reflects the drowning of these sands by a lagoon which was frequently marine: it includes widespread claystone barriers. Further marine transgression produced a return to Rannoch-like conditions, and Zone $4 \mathrm{~A}$ (I. Tarbert) was deposited during retrogradational wave action. Zone 4B (u. Tarbert), not everywhere easily differentiated, includes better quality sand downflank.

The overall controls on reservoir sand distribution are considered to be: (i) accommodation space (principally a function of tectonism), (ii) timing and location of fluvial input (affects grain size), (iii) degree of wave activity (affects sorting), (iv) proximity to marine influence (tidal modification), and ( $v$ ) sea-level rises (flow barriers).

\subsection{Petrophysics}

Fieldwide consistent log analysis procedures were applied in a deterministic manner using the dual water methodology. Where feasible, log derived parameters were calibrated against core values.

A log derived total porosity was obtained from the density log accounting for grain density variations and hydrocarbon effects. This log derived porosity was crossplotted against the overburden corrected core porosity and then linearly adjusted so as to give a best fit to the overburden corrected core porosity. The linear transformation coefficients were established for each well and individually for the water, oil and gas zones using constrained regression through the origin. 


\subsection{Relative Permeability Model}

A full SCAL program was carried out on the first well in the Brent-NI reservoir (34/8-8). 20 samples were tested under water and gas drive mechanisms, and the results suggested conformity and validity. The water-oil rel.perm. were based on high rate short-core reservoircondition displacement tests. The results indicated a $S_{\text {orw }}$ of $\approx 20 \%$. The existence of high residual oil saturations below the free water level suggests that there is little possibility of gravity-drainage-assisted recovery under water displacement. A more favourable displacement is anticipated under gas injection due to favourable gravity drainage. As a consequence, both gas displacement and gas centrifuge rel.perm. test results were used in establishing gas-oil rel.perm. curves ${ }^{9}$. The resulting gas-oil rel.perm. curves gave very low $S_{\text {org }}$, but with unfavourable mobilities to account for the gravity drainage process.

A segregated 3-phase oil rel.perm. model ${ }^{10}$ was used for the majority of this study. More detailed models including alternative 3-phase models ${ }^{11,12}$ and gas rel.perm. hysteresis were studied under the different drive mechanisms. It was determined from this evaluation that most of the displacement was 2-phase, which gave similar results for all the different 3-phase models.

\subsection{PVT Analysis and Fluid Characterization}

Two distinct fluid types have been discovered in the reservoir. The first well (34/8-8) discovered a "blackoil" with $R_{s}=180$ (single stage) and $\rho=0.85$. The second (34/8-10S) and third (34/8-11) wells found a "slightly volatile-oil" with $R_{s}=310$ and $\rho=0.82$. The two different fluids were determined to be separated by a sealing fault running north-south through the centre of the reservoir (Figure 1). Three well tests confirmed the fluid distribution. The fluid barriers are expected to break down and communicate when moderate pressure differences develop across them.

PVT studies were carried out on both fluid types and formed the basis for an EOS characterization. A single EOS model was developed to represent both fluid types as the fluids are expected to commingle during production because of "leaky" faults. The Peng-Robinson EOS $^{13}$ (modified 79 version) with 16 components was used to represent the fluid systems. The $C_{10+}$ fraction of the fluid compositions was split according to the Whitson Gamma distribution ${ }^{14}$ and the component critical properties were based on correlations from Twu ${ }^{15}$. The EOS model was developed through regression against the PVT experimental data. The critical temperatures and pressures, binary interaction coefficients and Gamma distribution parameters were used as the regression parameters in obtaining a satisfactory match to the pressure-volume data.

For compositional simulation of gas and WAG injection schemes, it was important not only to have the PVT behaviour correct but also the miscibility characteristics of the dual fluid system. To achieve this, the MMPs determined from slim tube testing were matched with the EOS model: the MMPs of the black-oil and slightly volatile-oil are 485 and 405 respectively, and the binary interaction coefficients between the heaviest component and other key components were adjusted in order to achieve a match to these MMPs. Finally the EOS model was pseudoized from 16. to 10 components to reduce the computational requirements. This final 10-component EOS model predicted the critical fluid properties, including MMPs, within $4 \%$ and the overall fluid properties within $10 \%$. The same EOS model was also used to generate the PVT properties for black-oil simulation.

\section{Reservoir Modelling}

\subsection{Cross-Sectional Modelling}

\subsubsection{Cross-Sectional Model}

The first stage of evaluation of the Visund Brent-NI reservoir was a detailed cross-sectional study. A model like this is often used to determine the optimal drive mechanism. The study was made following the drilling of the 34/8-8 well and was therefore based primarily on data from this well. The 7 stratigraphic zones formed the basis for the model layering. The zone average petrophysical properties were kept constant horizontally. To reduce numerical dispersion with the compositional simulation, the zones were arbitrarily subdivided into 45 equal-interval sub-layers. Horizontal permeability was also refined to match the well data for each of the $\mathbf{4 5}$ sub-layers. The $k_{V} / k_{H}$-ratio was based on the geometric averages for the 7 zones, without further refinement. An additional vertical transmissibility multiplier of 0.001 was placed in Zone $3 B$ to account for anticipated poor vertical communication.

The 2-D model was given a dip similar to that of reservoir (i.e. $7^{\circ}$ ) and was truncated to model the top erosional surface at a depth giving an oil column of $\approx$ $90 \mathrm{~m}$, which is the maximum in the reservoir. For water and downdip WAG injection, an injector was placed downflank and the producer was located in the dip parallel orientation along the erosional surface such that it penetrated the majority of the Brent zones. Under gas and updip WAG injection, a vertical injector was placed close to the erosional edge penetrating only the lower Brent zones. Because of the existence of a residual oil column below the FWL, a horizontal producer could be located very close to the FWL without being severely restricted by water cresting. Again, the producer was located in a dip parallel orientation cutting through the entire Brent sequence.

The model was initialized with both fluids for simulation under the four drive mechanisms, and run with full pressure maintenance at 434 bar, to maintain miscibility with the volatile oil under gas injection. The updip WAG was run on a $1: 1$ injection ratio with the injection cycling bi-monthly, while the downdip WAG had a 2:1 WAG ratio ( 4 months water injection followed by 2 months of gas). These WAG ratios were optimized on the 3-D heterogeneous model (Section 3.3) and may not be optimal for the 2-D model. 


\subsubsection{Simulation Results}

The initial runs using a black-oil simulator gave approximately the same recovery for all four displacement alternatives, see Table $1 \mathrm{a}$. where the ranking of the 4 mechanisms is also depicted, assuming $2 \%$-units as significant.

Table 1a. Summary of black oil simulation results.

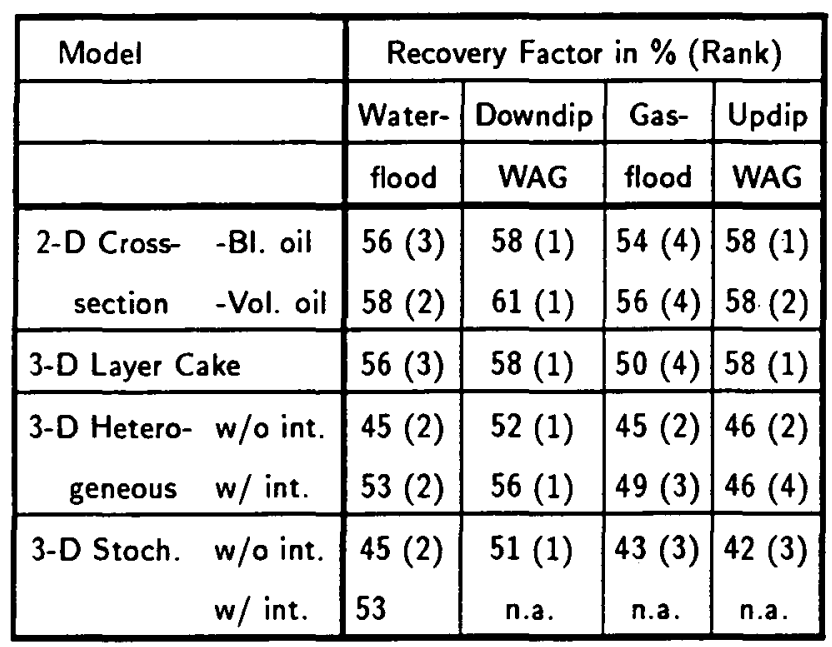

Table 1b. Summary of compositional sim. results.

\begin{tabular}{|c|c|c|c|c|}
\hline Model & \multicolumn{4}{|c|}{ Recovery Factor in \% (Rank) } \\
\hline & Water- & Downdip & Gas- & Updip \\
\hline & flood & WAG & flood & WAG \\
\hline 2-D Cross- -BI. oil & $56(4)$ & $59(3)$ & $62(2)$ & $64(1)$ \\
section -Vol. oil & $58(4)$ & $62(1)$ & $60(2)$ & $60(2)$ \\
\hline 3-D Layer Cake & $56(1)$ & $57(1)$ & $57(1)$ & $56(1)$ \\
\hline 3-D Hetero- w/o int. & $45(2)$ & $49(1)$ & $44(2)$ & $43(4)$ \\
geneous w/ int. & $53(1)$ & $52(3)$ & $54(1)$ & $44(4)$ \\
\hline 3-D Stoch. w/o int. & $46(1)$ & $44(2)$ & $40(4)$ & $42(3)$ \\
\hline
\end{tabular}

The recoveries all increased with the oil fluid volatility, more so under the downdip than with the updip injection schemes. Both EOR schemes improved recovery through an improved vertical sweep. In all cases displacement was governed by the highly permeable Zone $3 \mathrm{~A}$ (Etive). For the updip schemes the final residual oil saturation in these sands was $<10 \%$ due to favourable gravity drainage in this high quality formation. The upper Tarbert (Zone 4B) and lower Rannoch (Zone 1B) improved in recovery with the EOR schemes while the Etive recovery remained the same. The second injection phase tended to gravity segregate and sweep zones that were unswept by the primary injection phase. The performance profiles for the four mechanisms, assuming black-oil fluid properties, are shown in Figure 2.

Under compositional simulation, different displacement behaviour of the different mechanisms became evident. The updip schemes showed a significant increase in recovery over the black-oil simulation for the "black-oil" fluid. This indicates that even though full miscibility was not achieved, a high level of mass exchange

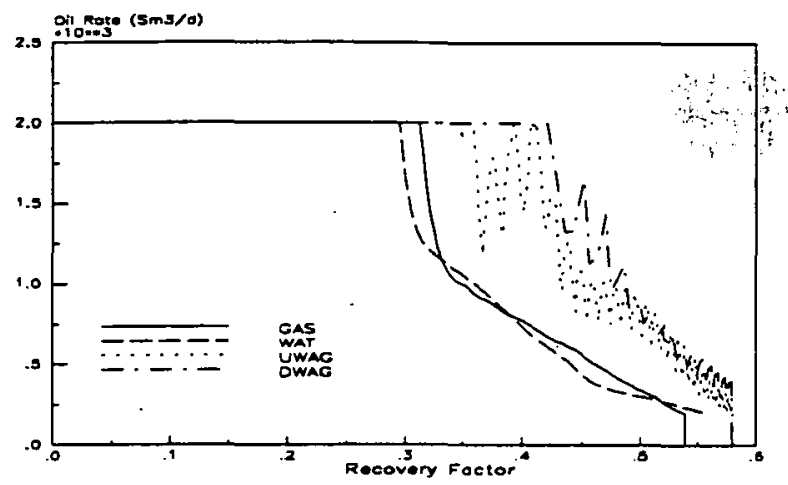

Figure 2. Production profiles for the four dispacement mechanisms under black-oil simulation in the 2-D model.

was occurring between the oil and the injected gas enough to reduce the residual oil saturation in the low permeability zones. When the same updip schemes were simulated with the volatile oil, lower recoveries were achieved. This is because the amount of gravity segregation and vertical cross-flow was reduced when the gas became miscible with the oil, resulting in a lower vertical sweep than was achieved with the immiscible fluid. The Etive zone has a high recovery through gravity drainage and/or miscibility. This tradeoff between local displacement efficiency (miscibility) and vertical sweep efficiency has also been reported in other studies ${ }^{16}$ for synthetic heterogeneous permeability fields.

For downdip drive mechanisms the results were more similar to those obtained from the black-oil simulation. The volatile-oil gave an improved recovery over the black-oil fluid system and the EOR scheme showed: a higher recovery than the primary water injection. The displacement for these schemes was controlled more:by the fluid mobilities, with the exception of the downdip WAG with the volatile oil which also benefited from the fluid miscibility.

\subsection{3-D Layer-Cake Modelling}

\subsubsection{3-D Layer-Cake Model}

Following the discovery of the volatile-oil with the 34/8$10 S$ well it was recognized that reservoir evaluation would have to be completed on the field scale to capture structural complexity and the commingling of fluidtypes during production. The structural model was subdivided and mapped on the 7 stratigraphic sequences. The petrophysical properties were likewise contoured on the 7 zones. The layer-cake model was further refined vertically to 18 equal-interval layers in a manner similar to the cross-sectional model. 18 layers were determined to be sufficient to resolve numerical dispersion under compositional simulation.

The permeability model was refined to 18 layer heterogeneity while maintaining the 7 zones contoured averages. The $k_{V} / k_{H}$ model was the same as in the crosssectional model. The base Zone $3 \mathrm{~B}$ was now modelled as sealing because a difference in reservoir pressure was observed in the $34 / 8-11$ well. 
The faults segmenting the dual fluid system were modelled as leaky barriers which would communicate once a threshold pressure had been exceeded. This threshold is believed to be in the $2-20$ bar range, with the base case set at 10 bar. In addition to the threshold pressures the faults were given a horizontal transmissibility multiplier $=0.001$.

The reservoir required 3 production wells to achieve a sufficient areal sweep. For the updip injection schemes the injectors were located along the east and west upflanks and the producers were located in a dip parallel orientation along the central low. For water injection and downdip WAG, the producers were mainly located along the east and west highs.

\subsubsection{Simulation Results}

For the layer-cake model run using black-oil simulation the results were about the same as those reported for the 2-D model (see Table 1a), except for gas injection where the recovery was reduced to $\approx 90 \%$ compared to the $2-\dot{D}$ case. With the full field model the oil column thickness varies from 50 to $90 \mathrm{~m}$ which is insufficient in places to allow a stable displacement and thus reduces the vertical sweep under gas injection. The updip WAG compensates for this by improving the recovery in the Rannoch and. Tarbert formations.

When the model was run using compositional simulation the gas injection scheme increased in recovery in the same order of magnitude as with the 2-D model. The increase was due to a higher microscopic displacement efficiency in all the formations. The increase was seen to be higher in the volatile-oil region than in the black-oil region. As with the 2-D model, the updip WAG had a slightly lower recovery than the primary gas injection due to trade-off between microscopic and vertical displacement efficiencies. The downdip schemes showed only a small reduction in recovery compared to the black-oil simulation results.

\subsection{3-D Heterogeneous Modelling}

\subsubsection{3-D Heterogeneous Model}

The initial subdivision of the reservoir according to stratigraphic sequences satisfactorily isolates the major depositional packages but is insufficiently detailed for prescribing probable flow units for simulation purposes, because the zonal averages had been arbitrarily constructed, ignoring critical heterogenities visible in the core. To identify the critical heterogeneities, we used the abundant core permeability data, plotted alongside the graphic sedimentary log, as in Figure 3. The objective initially was, for each well, to group cognate values for $k_{H}$ and $k_{V} / k_{H}$ without reference to descriptions of sedimentary lithofacies or position within the stratigraphic sequence framework.

The most crucial improvement looked for in the 3$D$ heterogeneous model was the adequate isolation of barriers, baffles and high permeability streaks, which the equal interval approach had tended to average out. It was therefore essential to correlate the petro-facies.
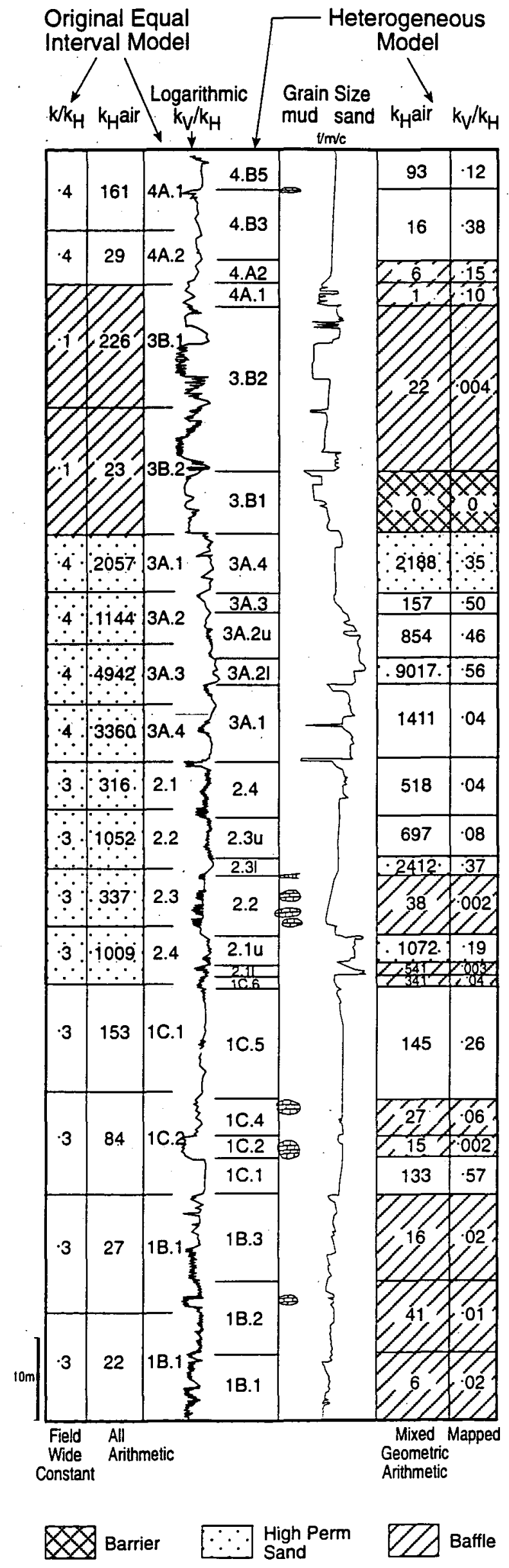

Figure 3. Equal interval vs. heterogeneous zonation schemes for Visund well 34/8-8. 


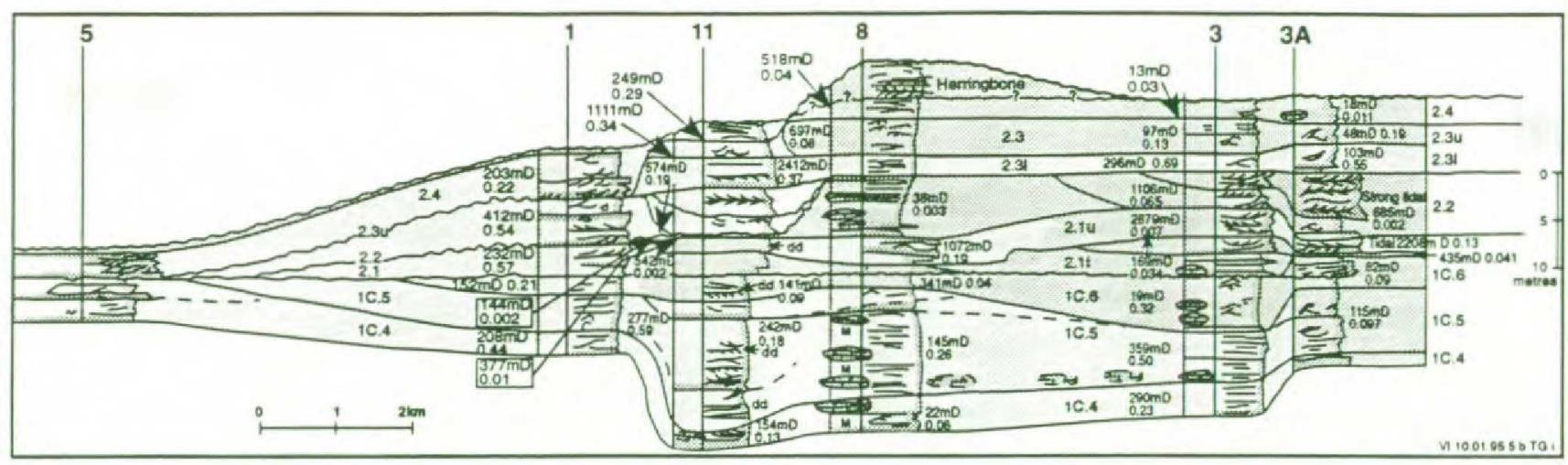

Figure 4. Visund Brent-NI reservoir zone correlation (Zones $1 \&$ 2).

using their position within the stratigraphic sequence shown in Figure 4.

Such a correlation portrays the tendency for barriers and high-perm streaks to be associated with particular levels in a sequence, but because of the relatively wide spacing of the wells it is clear that a deterministic approach cannot on its own predict horizontal aspects of heterogeneity adequately. Mapping our petrofacies by posting values at the wells enables a broad-brush picture to be painted which requires the subsequent selective addition of stochastic noise to form the most acceptable basis for simulation.

Depositional heterogeneity is thus the prime determinant on reservoir quality in Visund, but depth related diagenetic features become important particularly in the LSF petro-facies.

Interactive discussions within the team, involving the iteration of variously detailed correlations spanning different numbers of reservoir layers, led to a 26 layer model being selected. This number of layers captured the main vertical heterogeneities, and was well suited for reservoir simulation. These layers were of markedly unequal thicknesses compared to the 3-D layer-cake model (Figure 3.).

The preceding approach depends critically on regressions obtained from an analysis of the core permeability data, and on the handling of data from zones where plug coverage is inadequate. It is important to consider these aspects in more detail.

\section{- Permeability Variation}

Heterogeneity is scale dependent and horizontal and vertical permeability measurements at the core plug scale level, $(2.5 \mathrm{~cm})$ were used in this case study from extensive coring of the Brent Group. Two considerations were primary regarding permeability variation. (i) how to predict the permeability where core coverage was lacking and (ii) how to obtain the most representative zone averages.

Horizontal permeability prediction was made by identifying distinct facies, determining the core permeability to core porosity relationship for each facies and then using these correlations and the core calibrated, log derived total porosity to generate a log derived horizontal permeability. This synthetic permeability was constrained to equal the core horizontal permeability where it existed. This constrained permeability was used over intervals where the core coverage was scarcer than 2 plugs per metre. This procedure enabled a synthetic horizontal permeability to be generated in each interval where a facies type had been identified. Where the facies was unclear, a general Brent Group core permeability versus core porosity correlation was applied. Where core vertical permeability was lacking, it was computed from correlations with the core or synthetic horizontal permeability.

A good fit of $\log \left(k_{\text {core }}\right)$ versus $\phi_{\text {core }}$ implies that for a particular facies type there is a characteristic pore geometry within which the grain size varies in a regular manner with porosity. An example for the LSF facies is shown in Figure 5. Nonoverburden corrected porosities and permeabilities were used here as the effects of overburden were accounted for later during the reservoir simulation process. This was done using a SCAL derived linear correlation between permeability to oil at irreducible water saturation at reservoir conditions, and the core permeability to air at ambient conditions.

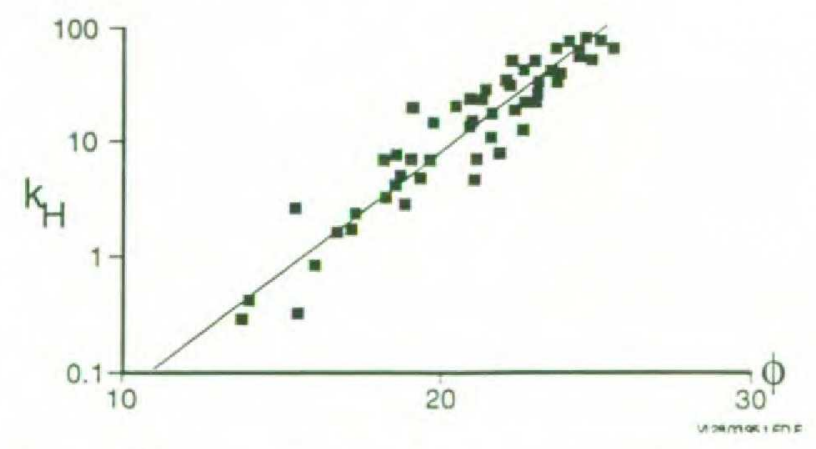

Figure 5. $\log \left(k_{\text {core }}\right)$ versus $\phi_{\text {core }}$ in the LSF facies.

Many types of permeability averages can be calculated. For the vertical permeability, the harmonic average within each reservoir layer was chosen as the most representative average. For the horizontal permeability, the choice was essentially between the arithmetic and geometric. The vertical permeability anisotropy is often quantitified via the $k_{V} / k_{H}$ ratio. This ratio was inspected plug by plug and used as an indicator of the degree of heterogeneity taking account of the facies type. From inspection of the magnitude and variation of the individual $k_{V} / k_{H}$ ratios and the character of the facies it was 
decided in a qualitative manner whether the arithmetic or geometric horizontal permeability average should be used for a particular reservoir zone.

\section{- Vertical Barriers}

In addition to the $k_{V} / k_{H}$ mapping, six layers had vertical transmissibility modifiers applied in order to capture the vertical barriers not included in the $k_{V} / k_{H}$ ratios. These transmissibility barriers were used to model internal and external shales and calcites which were not averaged into the horizontal or vertical permeability. The vertical transmissibility was reduced to zero over a limited extent depending upon its expected continuity.

Other than the layering and parameter modelling (26 layers) the heterogeneous simulation model was the same as the earlier Layer-Cake model. The fluid distribution, threshold pressures and general well locations all remained unchanged.

\subsubsection{Simulation Results}

The heterogeneous model showed a reduction in recovery under all drive mechanisms for both black-oil and compositional simulation (Table la \& $1 b$ ). The greater vertical heterogeneity caused more fluid fingering through the high permeability layers and consequently a much poorer vertical sweep efficiency. These recoveries and the vertical pressure profiles developed with the heterogeneous model are, however, much more consistent with those reported ${ }^{1-3}$ in mature Brent reservoirs. Under black-oil simulation the downdip WAG demonstrated a significant increase in recovery over the primary water injection scheme. This is again attributed to an improved vertical sweep and better attic oil recovery. The updip EOR scheme on the other hand showed no improvement in recovery over the primary gas displacement mechanism. The updip WAG improved the recovery in the lower Brent zones but reduced the upper Brent recovery due to a lower microscopic displacement efficiency with the introduction of the third phase.

To improve the zonal recoveries well interventions were utilized both to shutoff high watercut and GOR zones and to sidetrack producers into low recovery zones. For the downdip drive mechanisms an aggressive intervention program was adopted with two sidetracks and 3 zonal shutoffs. For the updip gas and WAG injection schemes it was found that a less aggressive intervention program (2 zonal shutoffs) was preferable, as the high microscopic displacement efficiency is sacrificed for improved vertical sweep. With well interventions the recoveries for all four displacement mechanisms under black-oil simulation improved. The large incremental increase in the downdip EOR scheme over the primary water injection, was reduced with the well interventions as would be expected. The updip EOR performed worse than the primary gas injection possibly due to unoptimal zonal shutoffs.

When the heterogeneous model was run with the compositional simulator and no well interventions the primary drive mechanisms performed in an equivalent manner to the black-oil simulation results. The gas injection scheme saw no significant increase in recovery

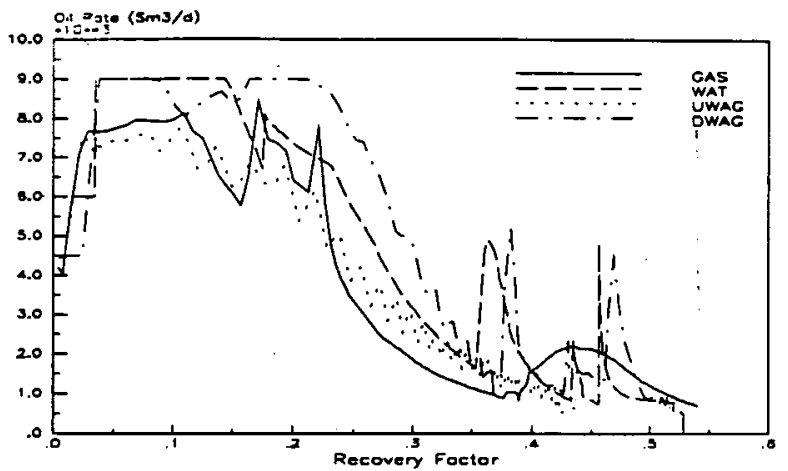

Figure 6. Production profiles for the four displacement mechanisms under compositional simulation with well interventions in the 3-D heterogeneous model.

as the Tarbert and Rannoch formations were poorly swept. The Etive formation on the other hand has a high recovery due to gravity drainage and/or miscibility, which resulted in similar recoveries for both blackoil and compositional simulation. A significant increase in the gas injection recovery was seen when the zonal shutoffs were included, which improved the displacement efficiency in the lower permeability zones. The updip WAG scheme performed equally poorly, with or without well interventions, because of the general reduction in microscopic displacement efficiency when the water phase is initroduced into the heterogeneous upper Brent.

The downdip WAG injection scheme saw a reduced vertical sweep under compositional simulation because of the miscible behaviour of the volatile oil. This trade-off between local displacement efficiency and gravity segregation and vertical crossflow, was verified by running the downdip WAG entirely with the immiscible blackoil fluid in-place. Under these conditions the vertical sweep improved and equaled that which was achieved under black-oil simulation. The recovery for the downdip WAG with well interventions fell short of that which was achieved by the primary water injection for the same reasons. A comparison of the production profiles for the four displacement mechanisms under compositional simulation is shown in Figure 6.

\subsection{3-D Stochastic Modelling}

The 3-D heterogeneous 26 layer model, with mapped properties in each layer, was developed to give a more correct representation of the heterogeneities in BrentNI. However, even this advanced model does not necessarily give a completely satisfactory representation of the reservoir, because: (i) there is an obvious uncertainty in the constructed maps, and (ii) the mapping is an interpolation/extrapolation procedure which by construction generates a too smooth image of the property variation. To investigate the effects of these factors, a simple stochastic simulation study was performed.

\subsubsection{3-D Stochastic Model}

\section{- Petrophysical Parameters}

The modelling was performed directly on the reservoir simulation grid. For each of the 26 layers, lateral 2D stochastic (log-) Gaussian fields were generated for: 

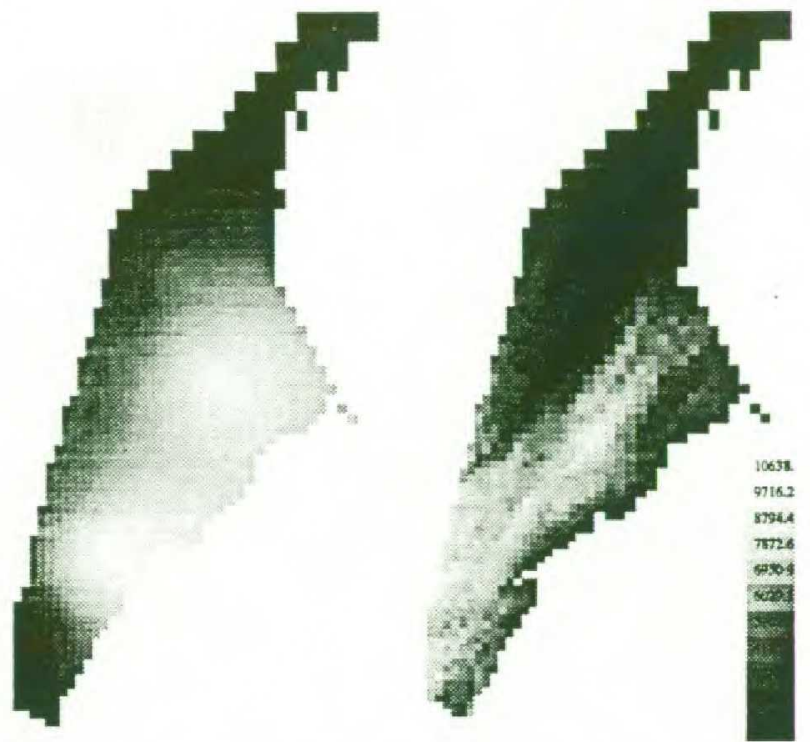

Figure 7 Deterministic horizontal permeability and one realization from the stochastic model for Zone $3 \mathrm{~A} .2$.

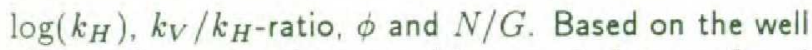
observations, a moderate positive correlation was found between all these four variables. The largest, 0.6 , was found between $\log \left(k_{H}\right)$ and $\phi$. No other correlation exceeded 0.35 . These correlations were used in the model.

The mean values for the 26 layers were taken from the deterministic model, to ensure that the stochastic and deterministic models varied around the same level. Similarly, the standard deviations for each layer in the stochastic model were chosen to give a slightly larger spread than in the deterministic model, to compensate for the smoothing in the deterministic model. The variograms were constructed by interpreting the hand-drawn maps which formed the basis for the deterministic model. The aim was to have stochastic realizations with the same overall visual appearance as the deterministic model, but with more variability and with its extremes possibly located elsewhere. The same variograms have been used for all the four parameters. Most of the 26 layers were regarded as vertically independent. However, a moderate vertical correlation was introduced in three layer sequences.

All the stochastic realizations were conditioned on the data from wells $34 / 8-8$ and $34 / 8-11$. To catch some of the trends in the data, two additional pseudo wells were introduced at the NE and SW model boundary. Data for these two wells were obtained by sampling the deterministic model.

Figure 7 shows the deterministic horizontal permeability as well as one realization from the stochastic model for zone 3A.2 (Upper Etive). The different appearence of the two modelling approaches can clearly be seen.

\section{- Stochastic Vertical Barriers}

To take the effect of additional vertical barriers into account, the deterministic model contains handdrawn shale barriers on some of the boundaries (Section 3.3.1).
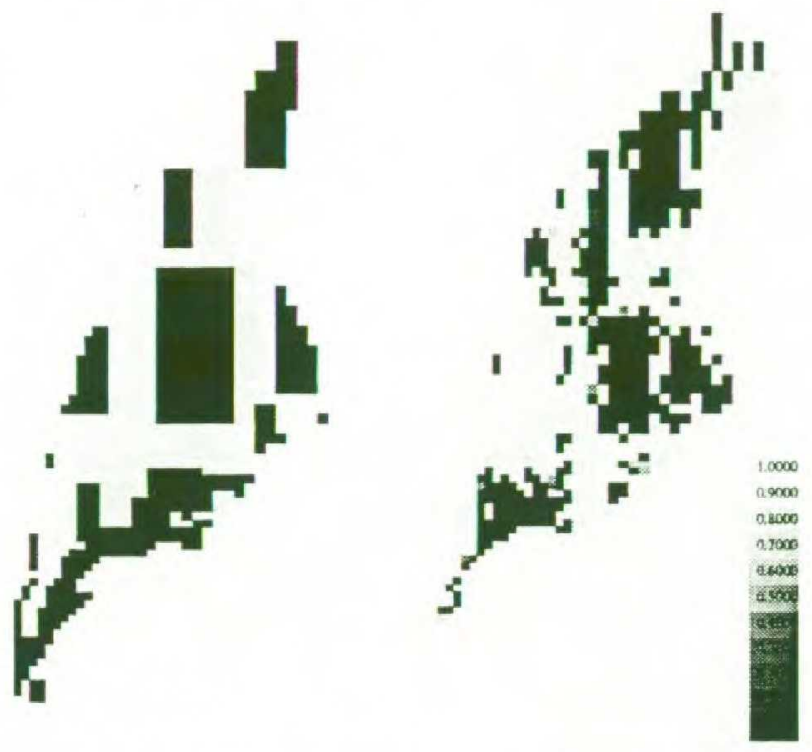

Figure 8 Deterministic and one stochastic realization of the barrier distribution at the Top 2.4 boundary.

Their extent and intensity vary from one boundary to an other. There are large uncertainties in the number, size and position of these shales. Therefore a stochastic shale model was constructed for five layer boundaries. The continuous barrier at Base Zone $3 \mathrm{~B}$ was maintained throughout. For a specific boundary, the transmissibility multiplier was modelled as a 2-D Gaussian field. truncated at 0 and 1 . The mean, standard deviation and variogram structure for the Gaussian fields were chosen to mimic the overall behaviour of the handdrawn base case barrier maps. The coverage and the overall size of the shales are about the same for the stochastic realizations as in the base case. However, due to the simple construction technique, the stochastic shales will tend to have a more "ragged" appearance.

All the stochastic realizations are conditioned on the presence or non-presence of shales in the wells 34/88 and 34/8-11. Figure 8 shows the deterministic and one realization of the barrier distribution at the Top 2.4 boundary.

\subsubsection{Simulation Results}

Five realizations were generated from the above combined stochastic petrophysics and barrier model. Each realization was submitted to the (otherwise) base case simulation model, with the different drive mechanisms. Only the non-intervention cases were examined as each realization would require a re-optimization of the intervention program.

For the black-oil simulation of the water injection scheme the mean recovery factor was found to be $99 \%$ of the base case deterministic model (Table 1a). The variability due to the stochastic nature of the models was also not very large, with the spread in recoveries (relative to base case) ranging from [95\% - 102\%]. For the downdip WAG the mean recovery was also $99 \%$ of the deterministic base case. however the spread in recoveries was reduced to [ $99 \%-100 \%]$. The updip injection schemes were somewhat more sensitive to the stochastic nature of the models with the mean recovery for 
gas and updip WAG (relative to the deterministic base) 96 and $93 \%$ respectively. The variability was, like the downdip cases, not very large but the range relative to the base case was lower for both gas injection [93\% $99 \%]$ and updip WAG [88\% - 98\%]. The five realizations were tested on the water injection scheme with well interventions which indicated that invention program was robust with a mean recovery of $99 \%$ and a range of $[98 \%-100 \%]$ of the base case.

Due to computational requirements of compositional simulation only a single realization (realization 3 ) was simulated for each of the different displacement mechanisms. Here again the compositional simulation results differed from those determined with black-oil simulation. The single realization fell outside the range predicted for both gas and downdip WAG injection. Under these two schemes the same reduced vertical sweep that was documented for the upper and lower Brent under compositional simulation of the heterogeneous model, is now apparent within the Etive formation for the stochastic model. Larger permeability contrasts within the upper Etive reduced the vertical sweep efficiency for the miscible volatile oil resulting in an overall lower recovery for this realization. The water injection and updip WAG injection schemes are not as severely affected as there is better vertical communication through the lower Brent which-allows-water-to-gravity-segregate-and-improve the recovery in these zones. Additional realizations are necessary to determine if these results are atypical.

\section{Model Comparisons}

\subsection{Downdip Iniection Schemes}

Under both black-oil and compositional simulation the 2-D and Layer-Cake models gave similar results showing the EOR scheme to perform marginally better than the primary drive mechanism. This however changed significantly with the more heterogeneous models. The black-oil simulation demonstrated a large upside with the downdip WAG over the water injection through an improved vertical sweep. When an aggressive well intervention program was adopted the benefits of the EOR scheme were reduced but still showed an upside potential in recovery. These results reflect what may be attainable with a fully immiscible system. For the Visund Brent-NI reservoir this is not the case and the gravity segregation and viscous crossflow behaviour were adversely affected by the miscibility of the volatile fluid system. This tended to reduce the vertical sweep and thus the advantages of the downdip WAG. For the stochastic model it even worsened. The recovery levels predicted by the four different models tended to remain similar, but only when an aggressive intervention program was included with the more heterogeneous models.

\subsection{Updip Injection Schemes}

The more homogeneous models (2-D and Layer-Cake) tended to overestimate the upside potential of the EOR scheme over the primary gas injection mechanism under black-oil simulation. The injected water phase tended to segregate to the lower Brent formations in the more homogeneous models allowing the gas to sweep the upper zones in an efficient manner. The restricted vertical communication in the heterogeneous models limited the amount of vertical crossflow of the water phase in the Upper Brent, which in turn reduced the local displacement efficiency in these zones. In addition the 2-D model did not reflect the structural importance on the gas injection recovery.

Under compositional simulation the EOR scheme tended to be equal to or worse than the primary gas injection under all the simulation models with the exception of the stochastic model. The reduced benefit of the updip WAG is due to both the higher recovery under gas injection due to miscible effects, and the impact of the aforementioned vertical restrictions to water crossflow in the heterogeneous models. The reduced recovery under gas injection in the stochastic model was due to reduced vertical sweep within the Upper Etive formation.

Based on the above observations and the ranking depicted in Table $1 a$ \& $1 b$, the prefered displacement mechanism for each level of reservoir description is summarized in Table 2.

Table 2 Optimal injection schemes

\begin{tabular}{|c|c|c|}
\hline Model & \multicolumn{2}{|c|}{ Optimal scheme(s) } \\
\hline & Black-oil & Compositional \\
\hline $\begin{array}{r}\text { 2-D Cross- -BI. oil } \\
\text { section -Vol. oil }\end{array}$ & D U & U \\
\hline 3-D Layer Cake & D U & W G D U \\
\hline 3-D Hetero- w/o int. & D & D \\
geneous w/ int. & D & D G \\
\hline 3-D Stoch. w/o int. & D & W \\
\hline
\end{tabular}

Legend: W-waterflood D - Downdip WAG

$$
\text { G-gasflood U-Updip WAG }
$$

\section{Conclusions}

Analysis of the results presented in this paper lead to the following conclusions:

- Detailed vertically heterogeneous reservoir models are needed to develop reservoir performance predictions and recovery levels, consistent with producing Brent Group reservoirs. Simpler homogeneous models overestimated the recovery levels and the benefits of the EOR.

- When injection alternatives are being studied on "realistic" heterogeneous models, well interventions must be considered, as this can be a more practical and cost efficient approach to improving overall recovery. On the Visund Brent-NI model the well intervention program had the effect of reducing the benefits of enhanced oil recovery.

- Proper treatment of the fluid behavior through compositional simulation was essential in establishing the 
most favourable drive mechanism and the appropriate recovery levels. Downdip WAG injection was notably affected by the trat deoff between miscibility (microscopic displacement efficiency) and gravity segregation (vertical sweep efficiency). This effect was magnified in the more vertically heterogeneous models.

- Even limited stochastic modelling of the petrophysics and vertical barriers was very important for quality assurance of deterministic models and to test the robustness of well intervention programs. It was found that these more heterogeneous permeability fields could both positively and negatively affect the zonal vertical sweep efficiencies, when proper treatment of the fluid behavior was accounted for.

- Our final, and most important conclusion, is: The level of reservoir description detail was less important in determining the optimal drive mechanism in black-oil simulation than in compositional simulation. When the fluid behaviour was properly accounted for through compositional simulation, the ranking of the displacement alternatives never reached an asymptotic state, which suggests that a decision on EOR should be delayed until the reservoir description is better understood through dynamic data acquisition.

\section{Nomenclature}

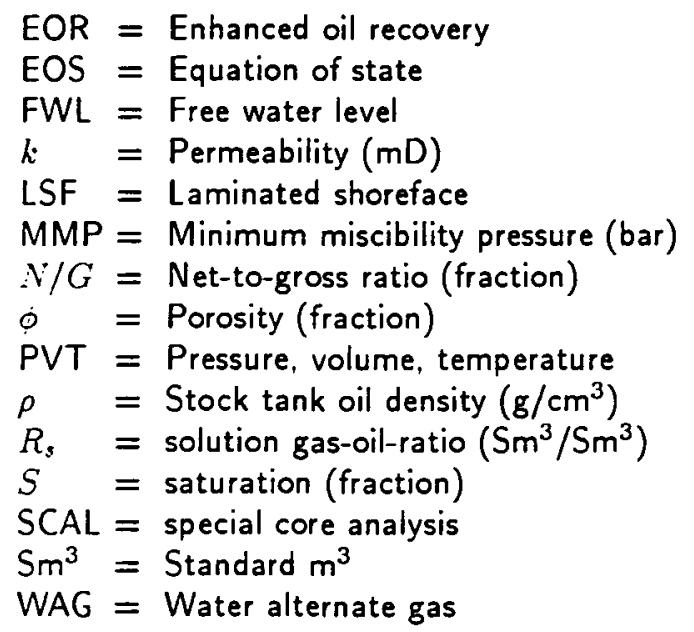

Subscripts

$H=$ Horizontal

org = Oil under gas displacement

orw $=$ Oil under water displacement

$V=$ Vertical

\section{Acknowledgement}

The authors would like to thank Norsk Hydro's management and our partners on the Visund field: Conoco Norway, Elf Petroleum Norge, Saga Petroleum and Statoil. for permission to publish this paper. Thanks are also due to our colleagues in the Visund project for their contribution, and to Norsk Hydro's drafting department for their excellent services.

\section{References}

1. Taylor, D.J. and Dietworst, J.P.A.: "The Cormorant Field, Blocks 211/21a, 211/26a, UK North Sea," Geol. Soc. Mem. 1991, No. 14, 73-81.

2. Petterson, O. et al:: "The Gullfaks Field," AAPG Mem. 1988, No. 54, 429-446.

3. Williams, R.R. and Milne, A.D.: "The Thistle Field, Blocks 211/18a and 211/19, UK North Sea," Geol. Soc. Mem. 1991, No. 14, 199-207.

4. Bryant, I.D. and Livera, S.E.: "Identification of Unswept Oil Volumes in a Mature Field by Using Integrated Data Analysis: Ness Formation, Brent Field, UK Northe sea,", Spec. Publ. EAPG 1991, No. 1. 75-88.

5. Haldorsen, H.H. and Damsleth, E.: "Stochastic Modeling," JPT April 1990, 404-412.

6. Damsleth, E. and Holden, L.: "Mixed Reservoir Characterization Models," paper SPE27969 presented at the 1994 University of Tulsa Centennial Petroleum Engineering Symposium, Tulsa, Aug. 29-31.

7. Stalkup, F.I. jr.: Miscible Displacement, SPE Monograph, SPE of AIME, Vol. 8, New York, 1984.

8. Brent Group, Morton, A.C. et al.(eds.), Geol. Soc. Lond. Spec. Publ. No. 61, London, 1992.

9. Stiles, J.H. jr. and Hutfilz, J.M.: "The Use of Routine and Special Core Analysis in Characterizing Brent Group Reservoirs, U.K. North Sea.". JPT June 1992, 704-713.

10. Eclipse 100 Technical Appendices, Intera Information Technologies Limited, Oxfordshire, 1994.

11. Stone, H.L.: "Estimation of Three Phase Relative Permeability", JPT 1970, Vol. 2, 214-218.

12. Stone, H.L.: "Estimation of Three Phase Relative Permeability and Residual Oil Data", JCPT 1973, Vol. 12, 53-61.

13. Peng, D.Y. and Robinson, D.B.: "A New TwoConstand Equation of State", Ind. Eng. Chem. Fund. 1976, Vol. 15, No. 1, 59-64.

14. Whitson, C.H., Anderson, T.F. and Soreide, 1.: " $C_{7+}$ Characterization of Related Equilibrium Fluids Using the Gamma Distribution," paper presented at the AIChE Spring Meeting, New Orleans, LA, March 610, 1988.

15. Twu, C.H.: "An Internally Consistent Correlation for Predicting the Critical properties and molecular Weights of Petroleum and Coal-Tar Liquids," Fluid Phase Equilibria 1984, Vol. 16, 137-150:

16. Pande, K.K.: "Effects of Gravity and Viscous Crossflow in Hydrocarbon Miscible Flood Performance in Heterogeneous Reservoirs," paper SPE 24935) presented at the 67th SPE annual Technical Conference and Exhibition, Washington D.C., Oct. 4-7, 1992. 